TOKYO J. MATH.

VoL. 3, No. 1,1980

\title{
On the Genus Fields of Pure Number Fields
}

\author{
Makoto ISHIDA \\ Tokyo Metropolitan University
}

In this paper, we shall investigate the genus fields of pure number fields $K=\boldsymbol{Q}(\sqrt[n]{a})$ with $a \in Z$. In the case of odd $n$, the genus fields of such number fields $K$ are explicitly determined in Fröhlich [1] and Ishida [3]. On the other hand, in the case of even $n$, the situations are somewhat complicated, as is expected from the genus theory of quadratic number fields. Here we shall show some partial results for the even degree cases and also give a new elementary proof for the odd degree cases. As for the definitions and some fundamental properties of genus fields, see Ishida [3].

\section{§ 1. Preliminaries.}

Let $K=\boldsymbol{Q}(\sqrt[n]{a})$ with $a \in Z(a \neq \pm 1)$ be a pure number field, where $a$ has the property

$$
p^{v} \| a \Longrightarrow(v, n)=1
$$

for any prime divisor $p$ of $a$. Then, of course, the degree of $K$ over $\boldsymbol{Q}$ is $n$. Let

$$
n=q_{0}^{s_{0}} q_{1}^{s_{1}} q_{2}^{s_{2}} \cdots q_{t}^{s_{t} t} \quad\left(s_{0} \geqq 0 ; s_{1}, s_{2}, \cdots, s_{t}>0\right),
$$

where $q_{0}=2$ and $q_{i}(i=1,2, \cdots, t)$ are odd primes, and put

$$
K_{i}=\boldsymbol{Q}\left((\sqrt[n]{a})^{n / q_{i}^{s_{i}}}\right) \quad(i=0,1, \cdots, t) .
$$

Then we have

$$
K=K_{0} \cdot K_{1} \cdot K_{2} \cdot \cdots K_{t} \quad \text { (composite). }
$$

Now let $K^{*}$ be the genus field of $K$ and $k^{*}$ the maximal abelian subfield of $K^{*}: K^{*}=k^{*} K$. Also let $k^{(i) *}(i=0,1, \cdots, t)$ be the maximal abelian subfield of the genus field of $K_{i}$. Since the degrees of $K_{i}$ over $\boldsymbol{Q}$ are coprime to each other, we have 


$$
k^{*}=k^{(0) *} \cdot k^{(1) *} \cdot \ldots \cdot k^{(t) *} \quad \text { (composite) }
$$

(cf. Proposition 2 in [3]). We denote by $\zeta_{m}$ a primitive $m$-th root of unity. Then $k^{(i) *}$ is obtained as the composite of two abelian subfields $k_{1}^{(i) *}$ and $k_{2}^{(i) *}$ :

$$
\boldsymbol{k}^{(i) *}=\boldsymbol{k}_{1}^{(i) *} \cdot \boldsymbol{k}_{2}^{(i) *} \quad\left(\boldsymbol{k}_{1}^{(i) *} \cap \boldsymbol{k}_{2}^{(i) *}=\mathbf{Q}\right)
$$

(cf. Chapter 4 in [3]). Here

$$
\begin{aligned}
k_{1}^{(i) *}= & \prod_{\substack{p \mid a, p \neq q_{i}\\
}}\left\{\text { the subfield, of degree }\left(q_{i}^{s_{i}}, p-1\right)\right. \text {, of the cyclotomic } \\
& \text { number field } \left.Q\left(\zeta_{p}\right)\right\} \text { (composite) }
\end{aligned}
$$

and, of course, the restrictions $p \neq q_{i}$ can be removed. On the other hand, as the degree $q_{i}^{a_{i}}$ of $K_{i}$ is a power of prime $q_{i}$,

$$
\begin{aligned}
& k_{2}^{(i) *}=a \text { subfield of the cyclotomic number field } Q\left(\zeta_{q_{i}^{r}}\right) \text { for } \\
& \text { some } r \in Z \text {. }
\end{aligned}
$$

Considering the ramification indices of $q_{i}$ in $k_{2}^{(i) *}$ and $K_{i}$, we see easily that the degree $\left[k_{2}^{(i)}: Q\right]$ is a power of $q_{i}$. Therefore our problem is to decide $k_{2}^{*}$ in special cases $n=q^{s}$ ( $q$ is an odd prime) and $n=2^{s}$ respectively.

§2. The case $n=q^{\text {s }}$.

Let $q$ be an odd prime and let $K=Q(\alpha)$, where $\alpha=\sqrt[q]{\mathfrak{a}} \bar{a}$ and $\dot{a} \in Z$ has the property (*) for $n=q^{s}$ (cf. Fröhlich [1] and Ishida [3]).

Then $k_{2}^{*}$ is a subfield of $Q\left(\zeta_{q^{*}}\right)$ for some $r$ and the degree $\left[k_{2}^{*}: Q\right]$ is a power of $q$. So, for the unique subfield $k_{0}$, of degree $q$, of the cyclotomic number field $Q\left(\zeta_{q^{2}}\right)$, we see that

$$
\begin{aligned}
k_{2}^{*}= & \Longleftrightarrow \boldsymbol{Q}_{2}^{*} \not \supset k_{0} \\
& \Longleftrightarrow k_{0} K \text { is not unramified over } K .
\end{aligned}
$$

On the other hand, in the sense of class field theory, the absolute abelian field $k_{0}$ corresponds to the ideal group

$$
H_{q}=\left\{(a) \mid(a, q)=1, a^{q-1} \equiv 1\left(\bmod ^{\times} q^{2}\right)\right\}
$$

in $Q$, with defining modulus $q^{2}$. Accordingly, by the 'Verschiebungssatz', we see

$k_{0} K$ is unramified over $K \Longleftrightarrow$ for any number $\gamma$ in $K$, prime to $q,\left(N_{K / Q} \gamma\right)^{q-1} \equiv 1\left(\bmod ^{\times} q^{2}\right)$. 
Now we consider the three cases separately. Here we denote by $N$ the norm mapping $N_{K / Q}$. (For the cases (1) and (2), the same proofs are already given in [3].)

(1) $q \mid a$. By $(*)$, we have $q^{v} \| a$ with $(v, q)=1$ and so there are $x, y \in Z\left(1 \leqq x \leqq q^{s}-1, y \geqq 0\right)$ such that $v x=1+q^{s} y$. Then $\beta=\alpha^{x} / q^{y}$ is an integer of $K$ and $\beta^{a^{*}}=b \in Z$ with $q \| b$. Put

$$
\gamma=1+\beta \in K \text {. }
$$

Then we have $N \gamma=1+b$ and so

$$
(N \gamma)^{q-1} \equiv 1+(q-1) b \equiv 1-b \not \equiv 1 \quad\left(\bmod q^{2}\right) .
$$

(2) $q \| a^{q-1}-1$. Pat

$$
\gamma=\alpha \in K
$$

Then we have $N \gamma=a$ and so

$$
(N \gamma)^{q-1}=a^{q-1} \not \equiv 1 \quad\left(\bmod q^{2}\right) .
$$

(3) $q^{2} \mid a^{q-1}-1$. Note that, for $c \in Z$ with $(c, q)=1, c^{q-1} \equiv 1\left(\bmod q^{2}\right)$ is equivalent to $c \equiv d^{q}\left(\bmod q^{2}\right)$ for some $d \in Z$.

Lemma 1. There is an integer $u$ such that

$$
1+u^{q} \equiv y^{q} \quad\left(\bmod q^{2}\right)
$$

for any $y \in Z$. (Of course, then $u$ is prime to q.)

Proof. Suppose that, for any $x \in Z, 1+x^{q} \equiv y^{q}\left(\bmod q^{2}\right)$ with some $y \in Z$. Then, putting $S=\left\{x^{a}\left(\bmod q^{2}\right) \mid x \in Z\right\}$, we can define an injective mapping

$$
f: x^{q}\left(\bmod q^{2}\right) \longmapsto 1+x^{q}\left(\bmod q^{2}\right)
$$

of the finite set $S$ to itself. Then $f$ is bijective and so is $f^{q}=f \circ f \circ \cdots \circ f$. Accordingly we have $0^{q}\left(\bmod q^{2}\right)=q+x^{q}\left(\bmod q^{2}\right)$ for some $x \in Z$, which implies $q \mid x$ and so $q^{2} \mid q$. This is a contradiction.

In (3), we have $a \equiv t^{q}\left(\bmod q^{2}\right)$ for some $t \in Z$. Let $u$ be the integer in Lemma 1 and $v \in \boldsymbol{Z}$ a solution of $v t \equiv u\left(\bmod q^{2}\right)$. Put

$$
\gamma=1+v \alpha \in K \text {. }
$$

Then we have $N \gamma=1+v^{q^{8}} a$ and so

$$
N y \equiv 1+v^{q} a \equiv 1+v^{q} t^{q} \equiv 1+u^{q} \equiv y^{q} \quad\left(\bmod q^{2}\right)
$$


for any $y \in Z$. So we have

$$
(N \gamma)^{q-1} \not \equiv 1 \quad\left(\bmod q^{2}\right) .
$$

Hence, for any case, there exists an integer $\gamma$ of $K$ such that $\gamma$ is prime to $q$ and $\left(N_{K / Q} \gamma\right)^{q-1} \not \equiv 1\left(\bmod q^{2}\right)$.

This implies that $k_{0} K$ is not unramified over $K$ and so we have

$$
k_{2}^{*}=\boldsymbol{Q} \text {. }
$$

$\S$ 3. The case $n=2^{s}$.

Let $K=Q(\alpha)$, where $\alpha=\sqrt[2 s]{a}$ and $a \in Z$ has the property (*) for $n=$ $2^{s}$. Then $k_{2}^{*}$ is a subfield of the cyclotomic number field $Q\left(\zeta_{2}\right)$ for some $r$ and the degree $\left[k_{2}^{*}: Q\right]$ is a power of 2 . So we consider all the subfields, of $Q\left(\zeta_{2^{r}}\right)(r=0,1,2, \cdots)$, of degree $2^{0}=1,2,2^{2}, \cdots$. They are easily listed up and illustrated by dots explicitly in the following diagram.

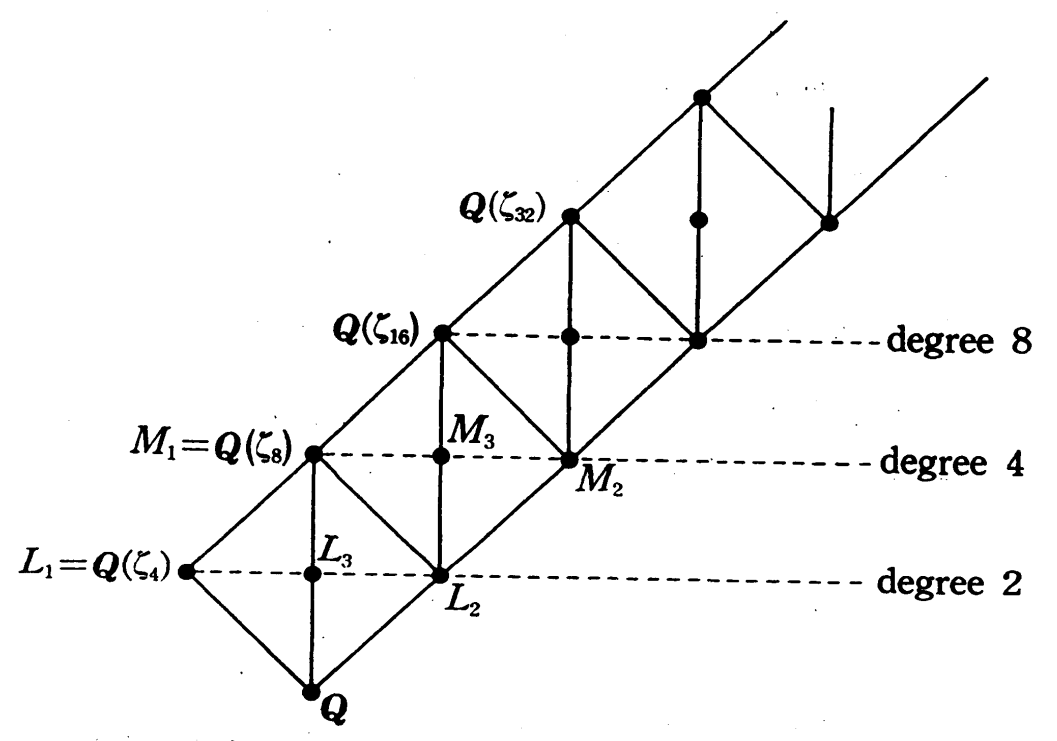

$$
\begin{aligned}
& L_{1}=Q(\sqrt{-1}), \\
& L_{2}=Q(\sqrt{2})=\text { the maximal real subfield of } Q\left(\zeta_{8}\right), \\
& L_{3}=Q(\sqrt{-2}), \\
& M_{2}=Q\left(\zeta_{18}+\zeta_{18}^{-1}\right)=\text { the maximal real subfield of } Q\left(\zeta_{18}\right), \\
& M_{3}=Q\left(\zeta_{18}-\zeta_{18}^{-1}\right) .
\end{aligned}
$$

That is, there are three such fields of degree 2 and also three such fields 
of degree 4. Moreover, in the sense of class field theory, they correspond to the ideal groups respectively, in $Q$, with defining modulus $16 p_{\infty}\left(p_{\infty}=\right.$ the infinite prime of $\boldsymbol{Q}$ ), as is shown in the following table.

\begin{tabular}{c|l}
\hline field & ideal gruop $\{(a)\}$ with $a \equiv$ \\
\hline \hline$Q\left(\zeta_{18}\right)$ & 1 \\
\hline$M_{1}=Q\left(\zeta_{8}\right)$ & 1,9 \\
\hline$M_{2}$ & 1,15 \\
\hline$M_{3}$ & 1,7 \\
\hline$L_{1}=Q\left(\zeta_{4}\right)$ & $1,5,9,13$ \\
\hline$L_{2}=Q(\sqrt{\overline{2}})$ & $1,7,9,15$ \\
\hline$L_{3}=Q(\sqrt{-2})$ & $1,3,9,11$ \\
\hline$Q$ & $1,3,5,7,9,11,13,15$ \\
\hline
\end{tabular}

Then, by the 'Verschiebungssatz', we see that, for example,

$M_{2} \subset k_{2}^{*} \Longleftrightarrow M_{2} K$ is unramified over $K$ (in narrow sense) $\Longleftrightarrow$ for any totally positive number $\gamma$ in $K$, prime to $2, N_{K / \boldsymbol{Q}} \gamma \equiv$ 1 or $15\left(\bmod ^{\times} 16 p_{\infty}\right)$.

Of course, if $k_{2}^{*} \neq Q$ i.e., $\left[k_{2}^{*}: Q\right]>1$ then $k_{2}^{*}$ contains at least one of $L_{i}(i=$ $1,2,3)$. Also, if $\left[k_{2}^{*}: Q\right]>2$ then $k_{2}^{*}$ contains at least one of $M_{i}(i=1,2,3)$. On the other hand, for even $a$, by (*), we have $2^{v} \| a$ with odd $v$ and so there are $x, y \in Z\left(1 \leqq x \leqq 2^{s}-1, y \geqq 0\right)$ such that $v x=1+2^{s} y$. Then $\beta=\alpha^{x} / 2^{v}$ is an integer of $K$ and $\beta^{2^{8}}=b \in Z$ with $2 \| b$. Writing $a=2^{v} c$, we have $b=2 c^{x}$ and so

$$
\begin{aligned}
& c \equiv 1(\bmod 4) \Longleftrightarrow b \equiv 2(\bmod 8), \\
& c \equiv 3(\bmod 4) \Longleftrightarrow b \equiv 6(\bmod 8) .
\end{aligned}
$$

For odd $a$, we put $\beta=\alpha(x=1)$ and $b=a$. Then the classical genus theory and the fact $Q(\sqrt{b}) \subset Q(\alpha)=K$ show that

$$
\begin{aligned}
& b(=a) \equiv 3(\bmod 4) \Longrightarrow k_{2}^{*} \supset L_{1}, \\
& b \equiv 2(\bmod 8) \Longrightarrow k_{2}^{*} \supset L_{2}, \\
& b \equiv-2(\bmod 8) \Longrightarrow k_{2}^{*} \supset L_{3} .
\end{aligned}
$$

Now put

$$
\gamma_{1}=A+\beta \quad(A \in Z) \quad \text { and } \quad \gamma_{2}=1+\beta^{2}+\beta \in K,
$$


where $A$ is so large that $\gamma_{1}$ is totally positive. Note that $\gamma_{2}$ is totally positive. Then we have $N_{K / Q} \gamma_{1}=A^{2^{2}}-b$.

LEMMA 2. We have $N_{k / Q} \gamma_{2}=1+b+b^{2}$.

Proof. Let $K^{\prime}=Q\left(\alpha^{2}\right)$. Then, as $\beta=\alpha^{x} / 2^{y}$ with odd $x$, we have $N_{K / R^{\prime}}, \gamma_{2}=\left(1+\beta^{2}+\beta\right)\left(1+\beta^{2}-\beta\right)=1+\beta^{4}+\beta^{2}$. Accordingly lemma is proved by the induction on $s$.

We consider the five cases separately. Here we denote by $N$ the norm mapping $N_{K / Q}$.

(1) $a=b \equiv 1(\bmod 4)$. Choose as $A$ a large integer divisible by 16 and so we have $N \gamma_{1} \equiv-a(\bmod 16)$. If $a \equiv 1(\bmod 16)$ then $N \gamma_{1} \equiv 15\left(\bmod 16 p_{\infty}\right)$ and $N \gamma_{2} \equiv 3\left(\bmod 16 p_{\infty}\right)$. Consequently the above table implies that $k_{2}^{*}$ contains none of $L_{i}(i=1,2,3)$ and so we have $k_{2}^{*}=Q$. In similar ways,

$$
\begin{aligned}
& a \equiv 5(\bmod 16) \Longrightarrow N \gamma_{1} \equiv 11, N \gamma_{2} \equiv 15\left(\bmod 16 p_{\infty}\right) \Longrightarrow k_{2}^{*}=Q, \\
& a \equiv 9(\bmod 16) \Longrightarrow N \gamma_{1} \equiv 7, N \gamma_{2} \equiv 11\left(\bmod 16 p_{\infty}\right) \Longrightarrow k_{2}^{*}=Q, \\
& a \equiv 13(\bmod 16) \Longrightarrow N \gamma_{1} \equiv 3, N \gamma_{2} \equiv 7\left(\bmod 16 p_{\infty}\right) \Longrightarrow k_{2}^{*}=Q .
\end{aligned}
$$

Hence we have

$$
k_{2}^{*}=Q \quad \text { if } \quad a \equiv 1(\bmod 4) .
$$

(2) $2 \mid a$ and $b \equiv 2(\bmod 8)$. Choose as $A$ a large integer such that $A^{2 s} \equiv 1(\bmod 16)$ and so we have $N \gamma_{1} \equiv 1-b(\bmod 16)$. If $b \equiv 2(\bmod 16)$ then $N \gamma_{1} \equiv 15\left(\bmod 16 p_{\infty}\right)$ and $N \gamma_{2} \equiv 7\left(\bmod 16_{\infty}\right)$. Consequently the above table implies that $k_{2}^{*}$ contains none of $M_{i}(i=1,2,3)$. On the other hand, as is remarked above, $k_{2}^{*}$ contains $L_{2}=Q(\sqrt{2})$. So we have $k_{2}^{*}=L_{2}$. In a similar way,

$$
b \equiv 10(\bmod 16) \Longrightarrow N \gamma_{1} \equiv 7, N \gamma_{2} \equiv 15\left(\bmod 16 p_{\infty}\right) \Longrightarrow k_{2}^{*}=L_{2} .
$$

Hence we have

$$
k_{2}^{*}=L_{2}=Q(\sqrt{2}) \quad \text { if } \quad b \equiv 2(\bmod 8) .
$$

(3) $2 \mid a$ and $b \equiv-2(\bmod 8)$. Choose as $A$ a large integer such that $A^{2^{s}} \equiv 1(\bmod 16)$. Then also in similar ways as in (2),

$$
\begin{aligned}
& b \equiv 6(\bmod 16) \Longrightarrow N \gamma_{1} \equiv 11\left(\bmod 16 p_{\infty}\right) \Longrightarrow k_{2}^{*}=L_{8}, \\
& b \equiv 14(\bmod 16) \Longrightarrow N \gamma_{1} \equiv 3\left(\bmod 16 p_{\infty}\right) \Longrightarrow k_{2}^{*}=L_{3} .
\end{aligned}
$$

Hence we have

$$
k_{2}^{*}=L_{3}=Q(\sqrt{-2}) \quad \text { if } \quad b \equiv-2(\bmod 8)
$$


(4) $a=b \equiv 3(\bmod 8)$. Choose as $A$ a large integer divisible by 16 and so we have $N \gamma_{1} \equiv-a(\bmod 16)$. Then also in similar ways as in (2),

$$
\begin{aligned}
& a \equiv 3(\bmod 16) \Longrightarrow N \gamma_{1} \equiv 13\left(\bmod 16 p_{\infty}\right) \Longrightarrow k_{2}^{*}=L_{1}, \\
& a \equiv 11(\bmod 16) \Longrightarrow N \gamma_{1} \equiv 5\left(\bmod 16 p_{\infty}\right) \Longrightarrow k_{2}^{*}=L_{1} .
\end{aligned}
$$

Hence we have

$$
k_{2}^{*}=L_{1}=Q\left(\zeta_{4}\right)=Q(\sqrt{-1}) \text { if } \quad a \equiv 3(\bmod 8) .
$$

(5) Then the remaining case is $a \equiv 7(\bmod 8)$. In this case, $\alpha+1=$ $\sqrt[2 s]{a}+1$ is a root of the Eisenstein polynomial $(X-1)^{2^{8}}-a$ with respect to 2. So 2 is totally ramified in $K=\boldsymbol{Q}(\alpha)$. Now we have the following

LEMMA 3. If $s=2$ and $K=Q(\sqrt[4]{a})(a \equiv 7(\bmod 8))$, then we have

$$
k_{2}^{*}=Q\left(\zeta_{8}\right)\left(=M_{1}\right) \text {. }
$$

Proof. By considering the degrees of $K$ and $M_{1}=Q\left(\zeta_{B}\right)$, it suffices to prove that $M_{1} K$ is unramified over $K$. Since we have $M_{1} K=$ $\left(M_{1}(\sqrt{a})\right)(\sqrt[4]{a})$ and

$$
\sqrt{a}-\frac{(\sqrt{a}+1)^{2}}{\left(\zeta_{8}+\zeta_{8}^{-1}\right)^{2}}=\frac{2 \sqrt{a-}(a+2 \sqrt{a}+1)}{2}=\frac{-(a+1)}{2} \equiv 0(\bmod 4),
$$

any prime ideal, dividing 2 , in $M_{1}(\sqrt{a})$ is unramified in $M_{1} K$ (cf. Hilbert [2]). Then it is easily seen that $M_{1} K$ is unramified over $K$.

Hence, for general case $s \geqq 2$ with $a \equiv 7(\bmod 8)$, it is shown that $\boldsymbol{Q}\left(\zeta_{8}\right) K$ is unramified over $K$ (in narrow sense) and so we have

$$
k_{2}^{*} \supset \boldsymbol{Q}\left(\zeta_{8}\right) \text {. }
$$

We note that $\boldsymbol{Q}\left(\zeta_{16}\right)$ is the only extension of $\boldsymbol{Q}\left(\zeta_{8}\right)$, of degree 8 over $\boldsymbol{Q}$, in the above diagram. On the other hand, choosing as $A$ a large integer divisible by 16 , we have $N \gamma_{1} \equiv-a(\bmod 16)$. Consequently if $a \equiv 7(\bmod 16)$ and $s \geqq 2$ then we have $k_{2}^{*} \not \supset Q\left(\zeta_{16}\right)$ and so

$$
k_{2}^{*}=M_{1}=Q\left(\zeta_{8}\right) \text {. }
$$

Of course, if $a \equiv 7(\bmod 16)$ and $s=1$ then we have $k_{2}^{*}=L_{1}=Q(\sqrt{-1})$. The cases $a \equiv 15(\bmod 16)$ and $s \geqq 3$ is still open.

\section{§4. Conclusion.}

Combining the results of $\S 1, \S 2$ and $\S 3$, we can determine the genus 
field $K^{*}$ of a pure number field $K=Q(\sqrt[n]{a})$, except the case $2^{3} \mid n$ and $a \equiv 15(\bmod 16)$. (In Theorem 7 of [3], the restrictions $p \neq q_{i}$ are unnecessary and must be removed (cf. §1).)

THEOREM. Let $K=\boldsymbol{Q}(\sqrt[n]{\bar{a}})$ with $a \in \boldsymbol{Z}(a \neq \pm 1)$ be a pure number field, where a has the property

$$
p^{v} \| a \Longrightarrow(v, n)=1
$$

for any prime divisor $p$ of $a$. Then the maximal abelian subfield $k^{*}$ of the genus field $K^{*}$ of $K$ is given as follows $\left(K^{*}=k^{*} K\right)$ :

$$
k^{*}=k_{1}^{*} \cdot k_{2}^{*} \quad(\text { composite }) \text {, }
$$

where

$$
\begin{aligned}
k_{1}^{*}= & \prod_{\substack{p \mid a \\
\text { the }}} \text { subfield, of degree }(n, p-1), \text { of the cyclotomic number } \\
& \text { field } \left.Q\left(\zeta_{p}\right)\right\} \quad \text { (composite) }
\end{aligned}
$$

and

\begin{tabular}{c|l|l}
\hline \multirow{2}{*}{$Q$} & $n$ & \multicolumn{1}{|c}{$a$} \\
\hline \multirow{2}{*}{$Q$} & odd & \\
\cline { 2 - 3 } & even & $a \equiv 1(\bmod 4)$ \\
\hline$Q(\sqrt{2})$ & even & $2^{v} \| a(v>0)$ and $a / 2^{v} \equiv 1(\bmod 4)$ \\
\hline \multirow{2}{*}{$Q(\sqrt{-2})$} & even & $2^{v} \| a(v>0)$ and $a / 2^{v} \equiv 3(\bmod 4)$ \\
\hline \multirow{2}{*}{$Q\left(\zeta_{8}\right)$} & even & $a \equiv 3(\bmod 8)$ \\
\hline & 2||$n$ & $a \equiv 7(\bmod 8)$ \\
\hline$?\left(\supset Q\left(\zeta_{8}\right)\right)$ & $2^{2} \mid n$ & $a \equiv 7(\bmod 16)$ \\
\cline { 2 - 3 } & $2^{2} \| n$ & $a \equiv 15(\bmod 16)$ \\
\hline
\end{tabular}

\section{References}

[1] A. Frölich, The genus field and genus group in finite number fields II, Mathematika, 6 (1959), 142-146.

[2] D. Hilbert, Uber die Theorie des relativquadratischen Zahlkörpers, Math. Ann., 51 (1898), 1-127 (Satz 5).

[3] M. IsHIDA, The genus fields of algebraic number fields, Lecture Notes in Math., 555, Springer, Berlin-Heidelberg-New York, 1976. 
Present Address:

Department of Mathematics

Faculty of ScIences

TOKYO METROPOLITAN UNIVERSITY

Fukazawa, Setagaya-ku, Tokyo 158 